\title{
Patología puerperal
}

\section{Puerperal pathology}

\author{
B. Bezares, O. Sanz, I. Jiménez
}

\section{RESUMEN}

El puerperio es el periodo desde el final del parto hasta la aparición de la primera menstruación. Las posibles patologías acontecidas en este tiempo son la causa más frecuente de mortalidad materna incluso en nuestro medio. La patología de la lactancia incluye el fallo de lactancia, grietas en el pezón, ingurgitación mamaria y mastitis puerperal.

La infección puerperal es una complicación obstétrica frecuente aunque las pautas de profilaxis han disminuido mucho la incidencia de esta patología.

La patología vascular del puerperio incluye cuadros de gravedad importante, representando una de las principales causas de mortalidad materna. Se incluye la trombosis venosa profunda y el tromboembolismo pulmonar como la complicación más grave de la primera.

No parece que la patología psiquiátrica sea más frecuente en el puerperio que en otra época de la vida, pero sí que se describen los cuadros relacionados con este periodo, como son el "blues", la depresión puerperal y la psicosis puerperal.

Por último, otros cuadros poco frecuentes, pero que pueden constituir una emergencia médica en el puerperio son el síndrome de Sheehan, la miocardiopatía periparto y la tiroiditis postparto.

Palabras clave. Puerperio. Infección puerperal. Trombosis venosa profunda. Tromboembolismo. Depresión puerperal.

\begin{abstract}
The puerperium is the period from the end of labour to the appearance of the first menstruation. The possible pathologies that can occur in this period of time are the most frequent cause of maternal mortality even in our setting. The pathology of lactation includes failure of breastfeeding, cracks in the nipple, mammary ingurgitation and puerperal mastitis.
\end{abstract}

Puerperal infection is a frequent obstetric complication although clinical guidelines for prophylaxis have considerably reduced its incidence.

The vascular pathology of the puerperium includes clinical features of great seriousness, representing one of the main causes of maternal mortality. These include deep vein thrombosis, of which pulmonary thromboembolism is the most serious complication

It does not appear that a psychiatric pathology is more frequent in the puerperium than in other periods of life, but the clinical features related to this period are described, such as "blues", puerperal depression and puerperal psychosis.

Finally, other clinical features which although infrequent can constitute a medical emergency in the puerperium are: Sheehan's syndrome, peripartum cardiomyopathy and postpartum thyroids.

Key words. Puerperium. Puerperal infection. Deep vein thrombosis. Thromboembolism. Puerperal depression.
Servicio de Ginecología y Obstetricia. Hospital Virgen del Camino. Pamplona

\section{Correspondencia:}

Blanca Bezares Pérez

Benjamín de Tudela, $47^{\circ} \mathrm{B}$

31008 Pamplona

E-mail: bbezares@hotmail.com 


\section{INTRODUCCIÓN}

El puerperio es el período que comprende desde el final del parto hasta la aparición de la primera menstruación. En este tiempo se desarrollan simultáneamente multitud de cambios fisiológicos en la mujer con la finalidad de retornar gradualmente al estado pregravídico y establecer la lactancia. Las posibles patologías acontecidas en este periodo son un motivo frecuente de consulta en urgencias, siendo además estos procesos la causa más frecuente de mortalidad materna, incluso en nuestro medio (Tabla 1) 1 . La hemorragia postparto no va a ser incluida ya que se va a desarrollar en el capítulo de la hemorragia postparto.

Tabla 1. Mortalidad materna en España*.

\begin{tabular}{lr}
\hline Causas obstétricas directas & $50 \%$ \\
Hipertensión (preeclampsia y eclampsia) & $30,76 \%$ \\
Embolia pulmonar & $23,07 \%$ \\
Hemorragia obstétrica & $23,07 \%$ \\
Otras causas... & $7,7 \%$ \\
Causas obstétricas indirectas & $42,30 \%$ \\
Patología cardiovascular & $42,30 \%$ \\
Patología neurológica & $36,30 \%$ \\
Patología infecciosa & $27,27 \%$ \\
Patología nefrourológica & $18,18 \%$ \\
Patología neoplásica & $9,09 \%$ \\
Causa desconocida & $7,69 \%$ \\
\hline *Extraída de De Miguel Sesmero, Causas de mortalidad materna \\
en España (modificado en 2002).
\end{tabular}

\section{PATOLOGÍA DE LA LACTANCIA}

Las ventajas de la lactancia materna están bien establecidas, tanto para el lactante como para la madre. En la $55^{\circ}$ Asamblea Mundial de la Salud, Ginebra 2002, se recomendó la lactancia materna exclusiva durante los primeros 6 meses de vida del bebé. Pero es habitual que se presenten dificultades, por lo que se debe promover una lactancia lo más precoz posible con una técnica correcta, lo que previene la formación de grietas y la consiguiente infección. Cuando se presentan complicaciones es nuestro deber intentar mantener la lactancia además del tratamiento oportuno.

El fallo de lactancia es la ausencia de producción de leche o la producción en cantidad insuficiente para el crecimiento del bebé. Hay que realizar primero una exploración para descartar causas anatómicas, como pezones planos, mamas tubulares o cirugías mamarias previas. Además se aplican medidas generales como repaso de la técnica correcta de la lactancia, asegurar una ingesta hídrica suficiente, instaurar pauta de alimentación a demanda, masaje suave en las mamas y calor húmedo antes de la toma, estimulación suave del pezón y areola y conseguir un entorno adecuado durante la lactancia. En estos casos es más importante la información que el tratamiento a prescribir. Las grietas en el pezón se manifiestan como dolor durante las tomas y pueden ser la puerta de entrada de las mastitis. Lo más importante es su prevención, realizando higiene del pezón antes y después de la toma. Se debe iniciar la toma por la mama menos afectada. Se pueden aplicar pomadas con analgésicos locales o corticoides de baja potencia si no se resuelve con estas medidas.

La ingurgitación mamaria se manifiesta como dolor y tensión mamaria muy intensos, 24-48 horas tras la aparición de la secreción láctea. La causa es una ingurgitación excesiva de venas y vasos linfáticos mamarios. Se trata mediante compresión, calor húmedo local, analgésicos y antiinflamatorios, vaciado de la mama de forma natural, y si no es posible de forma mecánica. La mastitis puerperal es la infección del parénquima glandular, tejido celular subcutáneo o vasos linfáticos de la mama. Se manifiesta con dolor, eritema, induración o masa, fiebre, leucocitosis con neutrofilia. Tiene una incidencia del 2 al 5\%, siendo el germen causante más frecuente el Stafilococus aureus (50\%), además escherichia coli, estreptococo y neumococo. Las vías de acceso son canalicular, linfática o hemática (en el contexto de una sepsis materna). Las formas de infección son del parénquima glandular (galactoforitis o absceso), del tejido celular subcutáneo (absceso premamario) o de los vasos linfáticos (linfangitis). Es importante distinguir la forma abscesificada de la no abscesificada (Fig. 1), ya que en el primer caso es necesario el tratamiento quirúrgico con incisión, desbridamiento y colocación de drenaje. El tratamiento médico requiere: analgésicos, antitérmicos, antiinflamatorios y antibióticos. Se inhibe la lactancia en caso de absceso o mal estado materno. La inhibición de la lactancia se 
realiza con cabergolina, medio comprimido cada 12 horas durante dos días. Las pautas de antibiótico son: cloxacilina $500 \mathrm{mg} / 6 \mathrm{~h}$, amoxicilina-clavulánico 875/125/8 h, clindamicina $300 \mathrm{mg} / 6 \mathrm{~h}$, cefalosporinas de $1^{\text {a }}$ ó $2^{\text {a }}$ generación, eritromicina $500 \mathrm{mg} / 6 \mathrm{~h}$ en pacientes alérgicas a la penicilina.

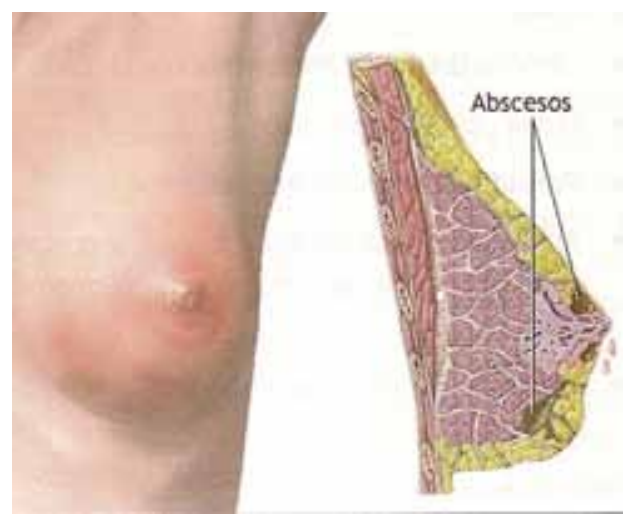

Figura 1. Abscesos mamarios.

Un galactocele es un quiste único localizado en la zona central de la mama, en la región ampular del conducto, bajo la piel. Se debe a la obstrucción de un conducto galactóforo que ocasiona la retención de leche. Se realiza diagnóstico diferencial con un proceso infeccioso por la exploración y la ausencia de signos inflamatorios. Se intenta su drenaje por presión y si no funciona se puede puncionar.

\section{INFECCIÓN PUERPERAL}

La infección puerperal es una complicación obstétrica frecuente. La importancia de este tipo de infecciones requiere un diagnóstico seguro, rápido y eficaz, así como un tratamiento antibiótico y ocasionalmente quirúrgico. A menudo las pacientes presentan síntomas iniciales de infección puerperal tras el alta, demandando la asistencia en el servicio de urgencias. Las pautas de prevención, cada vez más importantes, han disminuido la frecuencia de esta patología ${ }^{2}$.

La fiebre puerperal se define como temperatura igual o superior a $38^{\circ}$ al menos durante dos días y entre los días $2^{\circ}$ y $10^{\circ}$ tras el parto. Se puede deber a causas ge- nitales o extragenitales. Las genitales son: endometritis, infección de la episotomía o de la laparotomía de la cesárea, fascitis necrotizante, mastitis, tromboflebitis pélvica séptica. Dentro de las extragenitales se incluyen: complicaciones respiratorias, pielonefritis, absceso pélvico, tromboflebitis, tirotoxicosis, fiebre de origen medicamentoso.

La endometritis es la causa más frecuente de fiebre puerperal. En la mayoría de los casos se produce por vía ascendente tras la colonización microbiana cérvicovaginal. Los factores de riesgo son el parto por cesárea, el número de exploraciones previas, tiempo de bolsa rota mayor de 6 horas, duración del parto de más de 8 horas, presencia de meconio, alumbramiento manual, diabetes, corioamnionitis, heridas y desgarro del canal de parto ${ }^{3}$. La mayoría se corresponden con infecciones polimicrobianas. El diagnóstico se realiza por la presencia de fiebre, dolor uterino a la exploración, loquios malolientes y leucocitosis. Se requiere por tanto exploración, analítica y ecografía para descartar la presencia de restos y realizar el diagnóstico diferencial con las otras causas de fiebre puerperal. Está recomendada profilaxis antibiótica en los casos de parto por cesárea con una cefalosporina de $1^{\mathrm{a}}$ generación. El fundamento terapéutico de la endometritis es el manejo hospitalario de los antibióticos de amplio espectro que cubran fundamentalmente la presencia de los microorganismos de la flora vaginal, incluyendo anaerobios con producción de betalactamasas. Se recomienda tratamiento intravenoso hasta que la paciente permanezca afebril durante 48 horas. Los tratamientos orales no se recomiendan tras el tratamiento intravenoso salvo en presencia de estafilococos ${ }^{4}$. Pautas habituales son: clindamicina $900 \mathrm{mg} / 8 \mathrm{~h}$ + gentamicina $240 \mathrm{mg} / 24 \mathrm{~h}$; amoxicilina-clavulánico $1 \mathrm{~g} / 8 \mathrm{~h}$; ampicilina-sulbactam $3 \mathrm{~g} / 6$ $\mathrm{h}$; ticarcilina-clavulánico $3 \mathrm{~g} / 4 \mathrm{~h}$, cefoxitina $2 \mathrm{~g} / 6 \mathrm{~h}$. En caso de restos puerperales habría que realizar un legrado uterino bajo anestesia.

La infección de la episiotomía es una complicación poco frecuente, en torno al $1 \%$, disminuyendo su incidencia debido al perfeccionamiento de la técnica quirúrgica y la asepsia en el paritorio. Se producen en su mayoría por agentes polimicrobianos. A 
la exploración se aprecian signos inflamatorios locales, como dolor, eritema y edema. El tratamiento incluye curas locales, antibiótico de amplio espectro y en ocasiones desbridamiento de la herida si se observa la presencia de tejido necrótico, siendo entonces necesario añadir antibiótico que cubra Clostridium perfringens (penicilina G $6 \times 10$ millones de unidades cada 4 horas).

La infección de la herida quirúrgica aparece en un $2-5 \%$ de todas las cesáreas. Son factores predisponentes la cesárea urgente, tiempo quirúrgico prolongado, corioamnionitis, obesidad o malnutrición, anemia o alteraciones de coagulación e inmunosupresión. Se debe a gérmenes de la flora cutánea (estafilococo) o contenidos en la cavidad amniótica, procedentes del tracto genital inferior, siendo polimicrobianas en un $63 \%$, anaerobias en un $30 \%$ y aerobias en un $7 \%$. La profilaxis se realiza con una dosis de cefalosporina de $1^{a}$ generación vía intravenosa y la preparación de la piel con alcohol o clorhexidina. No hay evidencia de ninguna medida en concreto que disminuya la incidencia de la infección de la herida debida a la técnica quirúrgica ${ }^{5}$. El tratamiento depende de la severidad del cuadro infeccioso. En los casos más leves, sólo con celulitis no complicada, es suficiente con un solo antibiótico. Si hay líquido en la herida requiere drenaje o realizar cultivo del material. Si ese material es seroso suele ser suficiente con el drenaje; si es de características purulentas se debe realizar drenaje, limpieza y curas de la herida, estableciendo antibiótico según el cultivo, generalmente antibióticos de amplio espectro. Los casos de especial rapidez de desarrollo de la celulitis, con afectación sistémica y con amplia extensión de la infección se deben generalmente a infecciones por estreptococo del grupo A, y pueden acompañarse ocasionalmente de fascitis necrotizante requiriendo además del tratamiento antibiótico vía intervención quirúrgica. La fascitis necrotizante es una complicación poco frecuente, pero potencialmente muy grave. Se debe sospechar su diagnóstico ante una rápida ascensión de signos de celulitis en las primeras 24 horas, afectación del estado general de la paciente, desarrollo y extensión a tejidos adyacentes o presencia de crepitación. Causada por Estreptococo pyogenes, Clostridium perfringens o polimicrobiana. Requiere ingreso. Se recomien- dan antibióticos empíricos en el inicio del tratamiento con ampicilina-sulbactam; clavulánico-cicarcilina o cefotetan, hasta tener antibiograma. Puede ser necesario realizar ecografía o RNM para localizar la lesión y realizar desbridamiento quirúrgico y extirpación de todo el tejido necrótico.

La tromboflebitis pélvica séptica es una complicación puerperal infrecuente con mortalidad mínima que puede aparecer tras parto vaginal $(1 / 2.000)$ o tras endometritis postcesárea (1-2\%). Hay que sospecharla ante fiebre persistente de origen desconocido; se produce por la combinación de tres factores: hipercoagulabilidad, lesión vascular por infección o traumatismo y estasis venoso. Existe una forma aguda que se llama trombosis aguda de la vena ovárica, producida por un gran trombo en la vena ovárica. Se manifiesta por fiebre en picos acompañada de sudoración, taquicardia, dolor abdominal agudo y brusco. Se palpa una masa alargada y blanda en FID, también puede haber íleo paralítico, tromboembolismo pulmonar en $1 / 3$ de los casos, y a veces metástasis infecciosas. La forma crónica se manifiesta como fiebre de origen desconocido, se produce por múltiples microtrombos en los capilares venosos pélvicos. El estado general está menos afectado y no se acompaña de otros signos y síntomas. Se diagnostica generalmente por exclusión o al ver la correcta respuesta al tratamiento anticoagulante. Hay que realizar diagnóstico diferencial con un absceso o hematoma pélvico. El tratamiento es heparina a dosis terapéutica durante 7-10 días. Se añaden antibióticos de amplio espectro vía parenteral (los mismos empleados para la endometritis). Cuando falla el tratamiento médico puede estar indicada la ligadura de la vena o la venotomía con aspiración del coágulo si existe afectación por encima de las venas renales.

Posibles complicaciones respiratorias que causen fiebre son atelectasia o neumonía por aspiración bacteriana. La atelectasia se produce en las primeras 24 horas en mujeres sometidas a cesárea con anestesia general. Es la causa más frecuente de fiebre puerperal extragenital y se manifiesta por fiebre, taquipnea, taquicardia, disminución de ruidos respiratorios y crepitantes en bases pulmonares. Suele resolverse espontáneamente en 3-5 días y si no ocurre esto hay que pensar en la posibilidad de aspiración. 
Tanto la tromboflebitis venosa superficial como la profunda pueden originar fiebre puerperal. El diagnóstico viene dado por la exploración física (extremidad tumefacta y dolorosa, sensibilidad en la pantorrilla) y el tratamiento consiste en la administración de heparina y vendaje compresivo de la pierna. Otra causa de fiebre puede ser una pielonefritis, que suele aparecer tras la primera semana de puerperio y se manifiesta como bacteriuria con piuria, vómitos, fiebre en picos con escalofríos y dolor lumbar. Se diagnostica mediante sedimento, urocultivo y antibiograma. Requiere tratamiento antibiótico e ingesta hídrica adecuada. El absceso pélvico se caracteriza por fiebre intermitente a pesar de tratamiento antibiótico adecuado en una infección pelviana. En caso de ruptura puede originar una peritonitis. Si se forma en la parte anterior generalmente pueden ser drenados por ecografía y si lo hacen en la posterior (tabique rectovaginal) requieren drenaje quirúrgico.

La crisis de tirotoxicosis se manifiesta por fiebre, disfunción cardiovascular (taquicardia, arritmias), gastrointestinal (vómitos, diarrea, dolor abdominal) y alteraciones del sistema nervioso central (agitación, desorientación, estupor e incluso coma). Es potencialmente mortal por complicaciones sépticas o insuficiencia cardiaca. Analíticamente se observa leucocitosis, aumento de transaminasas e hipercalcemia. Requiere un tratamiento precoz con hidratación intravenosa, oxígeno, propanolol, antibioterapia y propiltiouracilo (inhibe la transformación de T4 en T3).

Puede darse también la fiebre de origen medicamentoso que es consecuencia de alergia a fármacos. Junto con la fiebre se pueden observar eosinofilia y exantema.

\section{PATOLOGÍA VASCULAR DEL PUERPERIO}

Durante el embarazo se producen una serie de cambios anatómicos y fisiológicos que van a favorecer y complicar el diagnóstico y manejo de los fenómenos tromboembólicos ${ }^{6}$. Por su gravedad van a constituir una de las principales complicaciones en el embarazo, parto y puerperio, representando una de las principales causas de mortalidad materna ${ }^{7}$. Debido a la escasez de estudios clínicos prospectivos, randomizados, no existe una clara evidencia clínica que soporte la eficacia y seguridad de las diferentes pautas de tratamiento en esta patología. La verdadera incidencia de trombosis venosa profunda (TVP) y de tromboembolismo pulmonar (TEP) es de entre un $0,26-0,7 \%$. Los factores asociados a un mayor riesgo de TVP son la paridad, cesárea, presencia de varices, edad mayor de 35 años, antecedentes familiares y personales de enfermedad tromboembólica y trombofilia. En estos casos está indicada la profilaxis antitrombótica. Tiene diferentes formas clínicas, siendo la más banal la flebitis superficial, que se manifiesta por dolor, eritema en un tracto venoso superficial con cordón palpable y aumento de la temperatura local. La trombosis venosa profunda se manifiesta por dolor, edema, aumento del diámetro de la extremidad afecta, signo de Homans positivo en un tercio de los casos; a veces hay espasmo arterial con con palidez y frialdad en esa extremidad y disminución de los pulsos periféricos. Puede haber fiebre y leucocitosis. El diagnóstico se confirma con eco doppler iniciando tratamiento precoz. Si el eco doppler es negativo pero existe fuerte sospecha clínica se puede tratar durante días. El tratamiento anticoagulane debe iniciarse precozmente y prolongarse en el tiempo. Son de elección las heparinas de bajo peso molecular (HBPM), habiendo distintas pautas. Las más usadas son dalteparina 200 ui $/ \mathrm{Kg}$ y enoxaparina $1,5 \mathrm{mg} / \mathrm{kg}^{8}$. Se añaden medidas generales como reposo con elevación de la extremidad afecta, aplicación de calor húmedo, vendas elásticas, ejercicios de flexión y extensión e iniciar deambulación cuando remita el dolor. Se debe continuar el tratamiento con anticogulantes orales hasta 3 meses tras el proceso agudo. La forma más grave es el embolismo pulmonar (EP). Es un proceso en el que un trombo se aloja en el árbol vascular provocando la obstrucción parcial o total del flujo arterial. La gravedad del cuadro depende de la extensión, siendo masivo cuando se produce la obstrucción de 2 ó más arterias lobares. En el 95\% de los casos el trombo proviene de una TVP de las extremidades inferiores.

El cuadro clínico es a veces muy inespecífico. El síntoma más común es la disnea (70\%), seguido de dolor pleurítico (66\%), 
tos (40\%) y hemoptisis (13\%). Es frecuente la sensación de angustia. El signo clínico más importante es la taquipnea, seguido de crepitantes y taquicardia. La clave del diagnóstico es la sospecha clínica. Hay que realizar además auscultación torácica, radiografía de tórax, electrocardiograma, ecografía transesofágica, analítica, gasometría. Para el diagnóstico de certeza se requiere gammagrafía pulmonar de ventilación-perfusión con una especificidad del $97 \%$ y una sensibilidad del 40\%; angiografía pulmonar, que si es negativa excluye el diagnóstico deEP; TAC helicoidal con una sensibilidad y especificidad que oscila entre un $86-95 \%$. Ante la sospecha fundada de EP se debe ingresar a la paciente en la UCI e iniciar el tratamiento anticoagulante, mientras se confirma el diagnóstico mediante gammagrafía o angiografía. La anticoagulación se realiza con heparina en perfusión intravenosa, con un bolo inicial de 10-15.000 UI, seguido de una perfusión continua de heparina en solución salina, de forma que se administran 15-20 $\mathrm{UI} / \mathrm{Kg} / \mathrm{h}$. Se añade oxigenoterapia, sedantes y analgésicos, aminofilina para el espasmo bronquial reflejo, fármacos inotrópicos si existe insuficiencia cardiaca derecha. A veces se requiere tratamiento trombolítico con estreptoquinasa o activador tisular del plasminógeno. Si los anticoagulantes fracasan o están contraindicados se realiza tratamiento quirúrgico, como tromboendarterectomía en pacientes con hipertensión pulmonar, embolectomía en pacientes con trombos en cavidades cardíacas derechas, ligadura o inserción de paraguas en la vena cava inferior en casos de embolización recidivante grave a pesar de la anticoagulación o en caso de hemorragia con riesgo vital. En general, usar métodos diagnósticos para el TEP es menor que el peligro de no tratarlo o de tratar algún paciente sintomático pero en realidad sin TEP.

\section{PATOLOGÍA PSIQUIÁTRICA DEL PUERPERIO}

No se sabe con certeza que la etapa perinatal aumente los trastornos psiquiátricos aunque en el primer mes postparto se considera de mayor riesgo. Conviene no olvidar que los síntomas de trastornos mentales pasan inadvertidos en el embarazo y puerperio o se clasifican como propios de esa situación. En nuestra labor está saber diferenciar la reacción emocional normal pasajera como resultado de un proceso de adaptación a la nueva situación, del hecho patológico que interfiere en su vida diaria y en el cuidado del niño para atenderle adecuadamente. Cualquier paciente que presente síntomas psiquiátricos de aparición reciente debe ser evaluada para descartar un consumo de sustancias psicoactivas, enfermedades médicas o neurológicas subyacentes. Para ello se realiza una anamnesis minuciosa, intentando describir todos sus síntomas, como alteraciones del comportamiento, llanto, fluctuaciones de conciencia, pérdida de apetito, pensamientos autodestructivos, detalles de la interacción entre madre e hijo, ansiedad excesiva, etc. Se realiza también una exploración médica que permita descartar enfermedades subyacentes.

Uno de los trastornos mentales más frecuente en el puerperio es la tristeza o "blues". Lo presentan el $40-80 \%$ de las puérperas, con síntomas depresivos que desaparecen en 7-10 días. No se considera una patología y no requiere tratamiento farmacológico, sólo tranquilizar y apoyar a la paciente y realizar un seguimiento para evitar que progrese. La depresión puerperal no parece ser un cuadro diferente al de la depresión mayor. Aparece en el 10-15\% de las puerperas ${ }^{10}$. Se manifiesta después de la segunda semana postparto. Se manifiesta en cansancio físico y psíquico excesivo, alteraciones del sueño, pérdida de interés y autoestima, anorexia, llanto, sensación de culpa. El tratamiento debe ser de inicio precoz e indicado por el psiquiatra. Son de primera elección la nortriptilina, imipramina, fluoxetina y paroxetina. La psicosis puerperal suele comenzar a las 2-3 semanas postparto. Es de inicio súbito, con desorientación témporo-espacial, inseguridad, confusión, insomnio, alucinaciones y delirios. De pronóstico favorable si se trata precozmente. Requiere ingreso, antipsicóticos en monoterapia como haloperidol o trifluoperazina.

\section{OTRAS PATOLOGIAS PUERPERALES}

Existen varios cuadros clínicos poco frecuentes en el postparto como la tiroiditis postparto, el síndrome de Sheehan, o la 
miocardiopatía periparto. La tiroiditis postparto se produce por una inflamación de origen autoinmune de la tiroides sobre una tiroiditis crónica subyacente. Aparece 1-6 meses tras el parto. En el $90 \%$ de los casos se produce una resolución paulatina a los 4-6 meses. Primero hay una fase de tirotoxicosis con destrucción de la glándula que origina liberación de hormona tiroidea 1-2 meses tras el parto, es autolimitada. Después hay una fase de hipotiroidismo a los 4-6 meses tras el parto, donde es frecuente la depresión. La mayoría de los casos no requieren tratamiento; éste se indicará según la clínica y no en base a los valores hormonales. El síndrome de Sheehan se produce por isquemia e infarto de la hipófisis debido a una hemorragia grave durante el parto, originando un panhipopituitarismo. Ocasiona problemas de lactancia y amenorrea, además de síntomas resultantes del déficit de cortisol y hormonas tiroideas. El tratamiento consiste en terapia hormonal sustitutiva. La miocardiopatía periparto es una causa infrecuente de fallo cardiaco en puérperas, con elevada mortalidad. Se manifiesta por la aparición de insuficiencia cardiaca en el último mes de embarazo o en los primeros 5 meses postparto sin causa identificable de fallo cardiaco. Se produce una disfunción sistólica de ventrículo izquierdo demostrada por criterios ecocardiográficos. La clínica puede ser inespecífica al comienzo, después se presenta con disnea, tos, ortopnea, disnea paroxística nocturna, hemoptisis, existiendo importante riesgo de tromboembolismo pulmonar y sistémico. Se diagnostica por la clínica, ecocardiograma, ECG, serología de Coxackie. El tratamiento incluye digoxina, diuréticos, restricción de sodio, betablo- queantes, profilaxis antitrombótica y en ocasiones, incluso trasplante cardiaco.

\section{BIBLIOGRAFÍA}

1. Sesmero JR. Mortalidad materna en España. Prog Obstet Ginecol 2002; 45: 524-534.

2. Smaill F, Hefmeyer GJ. Profilaxis antibiótica en la cesárea. (Revisión Cochrane traducida) En: Biblioteca Cochrane Plus, número 3, 2008. Oxford, update Software Ltd.

3. Newton ER, PRIHOda TJ, GibBs RS. Aclinical and microbial analysis of risk factors for puerperal endometritis. Obstet Gynecol 1990; 75: 402.

4. Fench LM, SAMIL FM. Antibiotic regimens for endometritis afeter delivery. Cochrane database Syst Rev 2002; CD001067.

5. Anderson ER, Gates S. Técnicas y materiales para pared abdominal en la cesárea. Revisión Cochrane traducida. En: Biblioteca Cochrane 4, 2005. Oxford: Update Sofware Ltd.

6. Rodger MA, Walter M, Wells PS. Diagnosis and treatment of venous thromboembolism in pregnancy. Best Pract Res Clin Haematol 2003; 16: 279-296.

7. Lewis G, editor. Why Mothers Die 2000-2002. Sixth Report of the Confidential Enquiries into Maternal Death. London: RCOG press; 2004.

8. ELDOR A. The use of low molecular weight heparin for the management of venous thromboembolism in pregnancy. Eur J Obstet Gynecol Reprod Biol 2002; 104: 3-13.

9. Darles-Bornoz JM, Gaillard P, Degiovanni A. Psiquiatría y embarazo: la madre y el niño. Enciclopedia Médico Quirúrgica Francesa. 2002. Editions Scientifiquies et Medicales Elsevier SAS, París.

10. Hendrick V, Altshueler L. Management of major depresión during pregnancy. Am J Psychiatry 2002; 159: 1667-1673. 
